\title{
POLLUTION, AGRESSION ET RADIOPROTECTION
}

\author{
Georges MARBLÉ \\ Président de la Société Française de Radioprotection
}

\section{I - INTRODUCTION}

Le titre et l'objet de cet exposé m'ont été inspirés par la lecture d'un numéro spécial de la revue Agressologie et d'une plaquette intitulée Nature et Homme.

Le numéro de la revue Agressologie, revue internationale de physiologie et de pharmacologie appliquées aux effets de l'agression, rassemble les communications faites au cours de plusieurs journées d'études organisées par l'Ecole d'Anthropologie dans le cadre de l'Année européenne pour la protection de la nature. Les bioclimats, la biométéorologie urbaine, les polluants atmosphériques, la pollution acoustique et les retombées radioactives constituent l'essentiel des sujets abordés au cours de ces journées.

La plaquette éditée par le Comité national et général d'action pour la sauvegarde de la vie et des équilibres biologiques réunit quelques articles généraux concernant la pollution et un article intitulé "Vers la fin, déchéance due aux rayonnements ionisants ». Le titre et quelques mots extraits de cet article :

"L'bumanité va pourrir de déchéance radioactive... il faut stopper cette mortelle industrie atomique... ou accepter d'en crever») ne laissent aucun doute sur l'attitude agressive de l'auteur vis-à-vis de toutes les activités dérivées de l'énergie nucléaire.

Pollution et agression, tєrmes très à la mode actuellement et surtout employés pour mettre en cause le développement industriel, en particulier nucléaire, ont-ils la même signification et ne peut-il y avoir de pollution limitée et contrôlée qui ne soit agressive?

La pollution radioactive doit-elle figurer au premier rang de toutes les sortes et formes de pollution, d'origine industrielle sans doute, mais également d'origines très diverses. Devons-nous accepter d'être sans cesse les victimes des accusations démagogiques de censeurs irresponsables ou devons-nous, au contraire, démontrer qu'aucun progrès technique ou scientifique n'est possible sans l'acceptation de certains risques, risques qui doivent être évalués de manière précise de façon à ce que puissent être mis en œuvre, pour les juguler et les domestiquer, les moyens de protection les plus adéquats.

Le développement industriel rapide que l'on observe, actuellement, dans les pays développés, s'accompagne de sources de nuisances les plus diverses qui

* Allocution prononcée à l'Assemblée générale de la Société Française de Radioprotection, le 2 juin 1972 .

RADIOPROTECTION, VOL. 7 - $\mathrm{N}^{\circ} 4$ 
constituent parfois un réel danger pour l'homme; mais ce danger doit être évalué, calculé, mesuré avec la rigueur et la précision qui sont de règle en radioprotection et il est absolument indispensable que, pour toutes les formes de pollution, soient mises en œuvre, par des techniciens compétents et responsables, des techniques élaborées et efficaces de protection. L'exemple et, pourquoi pas, le concours des techniciens de la radioprotection devraient permettre de faire en sorte que, dans tous les domaines, pollution ne soit pas synonyme d'agression.

Je vais maintenant dresser un rapide tableau des diverses sortes de pollution de l'environnement et j'essaierai ensuite de comparer les méthodes employées et les résultats obtenus dans les différents domaines et ce bilan sera, j'en suis sûr, favorable à la radioprotection.

\section{2 - POLLUTION ET POLLUANTS}

\section{I. Pollution Radioactive}

Les essais d'armes nucléaires et les rejets d'effluents provenant des installations nucléaires constituent les sources essentielles de pollution par des produits de fission ou d'activation. C'est un problème que nous connaissons tous bien, et je ne m'étendrai pas sur ce point.

Les risques d'accident de réacteur deviennent de plus en plus improbables, l'utilisation des radioéléments artificiels est rigoureusement contrôlée et les conséquences écologiques et biologiques des pollutions atmosphériques et marines sont tout particulièrement étudiées et surveillées.

L'industrie nucléaire ne doit pas toujours être mise en cause, et je citerai à ce propos l'article récent de BARTels paru dans Health Pbysics d'avril 1972 concernant la contribution volcanique à la pollution atmosphérique en ${ }^{10} \mathrm{Be}$, ${ }^{35} \mathrm{~S},{ }^{32} \mathrm{P}$ et ${ }^{22} \mathrm{Na}$.

Plusieurs millions de tonnes de chlore, de soufre, de fluor, de bore, de sodium, de potassium, de calcium sont libérés annuellement dans l'atmosphère par les éruptions volcaniques et des réactions nucléaires provoquées par les particules d'origine cosmique engendreraient les radioisotopes précités.

\subsection{Pollution ChimiQue}

Les polluants chimiques sont de natures très diverses et ne sont pas tous une conséquence du développement industriel.

Les oxydes de soufre, et tout particulièrement l'anhydride sulfureux $\mathrm{SO}_{2}$, sont très répandus dans l'atmosphère et très dangereux, en eux-mêmes, et par le fait qu'ils se transforment en acide sulfurique ou en sulfates. Ces produits de combustion proviennent des raffineries de pétrole, des centrales électriques, des combustions industrielles de toutes sortes. 70 millions de tonnes de $\mathrm{SO}_{2}$ sont rejetés par an dans l'atmosphère. 
L'oxyde de carbone provenant des gaz d'échappement des automobiles est dû à la combustion incomplète de l'essence. On estime que la quantité de CO libérée en une année dans l'atmosphère est de l'ordre de 180 millions de tonnes.

Le rejet dans l'air du gaz carbonique, produit normal de la combustion des composés organiques, se traduit par une augmentation progressive de la teneur de l'air en $\mathrm{CO}_{2}$ d'environ $\mathrm{I} \%$ par an.

Les gaz d'échappement des voitures contiennent également des oxydes d'azote et des hydrocarbures non brûlés. Certains de ces hydrocarbures sont concérigènes comme le benzo-3-4 pyrène et on les retrouve aussi dans les rejets des produits pétroliers provenant des raffineries ou de la vidange des soutes des navires pétroliers. On ne se rappelle que trop l'accident du Torrey Canyon et les méfaits de la " marée noire ».

L'emploi sans contrôle de grandes quantités de détergents, d'engrais, de pesticides, d'herbicides pose de sérieux problèmes. Les détergents les plus utilisés sont généralement des alkyl-aryl-sulfonates qui ne sont pas dégradés par les micro-organismes et sont des composés tensioactifs très nocifs. L'utilisation massive des engrais, en agriculture, se traduit par l'augmentation de la teneur des eaux en nitrates, nitrates qui peuvent se transformer en nitrites dans le tube digestif. Les insecticides sont très utilisés en agriculture, en élevage, dans les habitations pour détruire les parasites. Les insecticides et les fongicides sont également très employés dans l'industrie de la pâte à papier, des bois, des tissus. Or, les dérivés organo-chlorés comme le DDT ou l'HCH, les composés arsenicaux, les organo-mercuriels, la diphénylamine ou l'acétylaminofluorène sont toxiques et se retrouvent dans l'eau ou les aliments. Il en est de même pour les herbicides destinés à détruire les mauvaises herbes et qui sont soit des phytohormones, soit des chlorates de sodium ou de potassium.

Le problème des additifs alimentaires est aussi très préoccupant. En effet, dans le but d'améliorer l'aspect, la présentation, l'odeur, la saveur des aliments ou pour leur assurer une meilleure conservation, des composés chimiques les plus divers sont ajoutés en quantité non négligeable. Ce sont des antiseptiques tels que l'acide monobromoacétique, des antioxydants comme la thio-urée ou la diphénylène-diamine, des édulcorants et aromatisants comme la dulcine, le safrol, les éthers éthylique et propylique de l'aminonitrophénol. Pour blanchir les farines, on utilise du trichlorure d'azote et, pour colorer des pâtes alimentaires ou des margarines, on ajoute du paradiméthylaminoazobenzène. Il faut aussi signaler que certains emballages plastiques peuvent transférer aux aliments qu'ils contiennent des produits toxiques comme le phosphate de triorthocrésyle.

Je ne mentionnerai que pour mémoire la longue liste des composés chimiques utilisés dans l'industrie pouvant donner lieu à des intoxications professionnelles : le chlore, le phosgène, l'ammoniaque, l'hydrogène sulfuré, le benzène, les amines aromatiques, etc... et les maladies professionnelles bien connues comme le saturnisme, l'hydrargyrisme, la silicose, etc...

Les médicaments ne causent actuellement que peu d'accidents, si l'on excepte le cas malheureux de la thalidomide, car leur usage est rigoureusement contrôlé, mais leur emploi en élevage, en particulier celui de certaines hormones, doit faire l'objet d'une surveillance spéciale. Il faut cependant noter que, bien qu'une réglementation sévère régisse l'usage des drogues, on assiste à une recrudescence de l'usage abusif des tranquillisants et des psychotropes comme le L.S.D. La

vOL. $7-\mathrm{N}^{\circ} 4$ 
mise en circulation de ces produits, en dépit de la législation et de la réglementation, peut être considérée, d'une certaine manière, comme une nouvelle forme de pollution.

\subsection{Pollution biologique}

La pollution biologique par les bactéries, les virus, les insectes, les animaux ou végétaux parasites relève de l'hygiène, de la bactériologie, la virologie, l'épidémiologie, disciplines essentiellement médicales. Il existe également une forme de pollution, appartenant au domaine de l'allergologie; il s'agit de la pollution liée aux allergènes. Cette pollution peut être due aux pollens, aux moisissures atmosphériques ou à des pneumoallergènes (ricin, oléagineux, céréales ou substances chimiques).

\subsection{Pollution physiQue}

Si l'on classe à part la pollution radioactive, il existe cependant divers types de pollution physique, je veux parler de la pollution acoustique et de la pollution thermique. Les nuisances dues au bruit, au froid ou à la chaleur ont été très étudiées, des normes ont été établies et des mesures de protection ont été définies.

Je placerai également dans ce groupe la pollution par les poussières, les fumées et les microparticules.

A ce propos, je voudrais indiquer que l'industrie souvent incriminée comme principal agent de pollution n'est pas seule en cause. Il ressort, en effet, d'un rapport récent de l'Organisation Mondiale de la Santé que la concentration moyenne des fumées est beaucoup plus élevée dans plusieurs villes de l'Inde que dans un grand nombre de villes plus industrialisées situées dans d'autres régions. En 1970, les concentrations moyennes mesurées à Delhi, Calcutta et Kanput ont été respectivement de $60,34 \mathrm{I}$ et $544 \mu \mathrm{g} / \mathrm{cm}^{3}$, la teneur en $\mathrm{SO}_{2}$ étant relativement faible : $4 \mathrm{I}, 33$ et $\mathrm{r} 2 \mu \mathrm{g} / \mathrm{m}^{3}$, donc forte pollution due à des fumées à faible teneur en soufre, alors qu'au Royaume-Uni la teneur en fumée est passée de $170 \mu \mathrm{g} / \mathrm{m}^{3}$ en 1958 à $60 \mu \mathrm{g} / \mathrm{m}^{3}$ en $\mathrm{i} 968$. Il apparaît donc que la pollution atmosphérique par les foyers domestiques est loin d'être négligeable.

\subsection{Pollution psychologiQue}

Il existe également une forme de pollution très dangereuse, je veux parler de la pollution psychologique qui peut être illustrée par les deux exemples suivants :

- une campagne publicitaire, faisant appel aux techniques modernes audiovisuelles et vantant les qualités d'un produit miracle, conduit une grande partie du public à l'employer, sans contrôle ni mesure. Il en est ainsi de l'utilisation des cosmétiques et produits " de beauté " de toutes sortes et le XIVe Congrès international de dermatologie qui s'est tenu récemment à Venise a fait le bilan de tous les accidents cutanés d'origine allergique dus à l'usage incontrôlé des 
crèmes, gelées, etc... et les dermatologues se sont élevés contre l'agressivité publicitaire qui serait d'après eux responsable de ces accidents;

- le deuxième exemple que je voudrais citer est l'impact produit sur le public par la publication répétée de contre-vérités tendant à exagérer les risques... de la contamination radioactive, par exemple, en cherchant à faire peur par des formules à l'emporte-pièce telles que «l'humanité va pourrir de déchéance radioactive... il faut stopper cette mortelle industrie atomique... ou accepter d'en crever "). Il serait, bien sûr, tout aussi dangereux de minimiser systématiquement les risques.

Cette énumération des divers types de pollution montre, d'une manière évidente, que le problème de la contamination radioactive n'est pas l'unique préoccupation qui doit prévaloir dans la protection de l'environnement, d'autant plus qu'une étude détaillée et approfondie des effets biologiques des différents polluants pourrait aisément montrer qu'elle n'est pas non plus la plus dangereuse, tout au moins dans la mesure où les règles de protection qui sont actuellement en usage restent strictement appliquées.

\section{3 - PROTECTION CONTRE LA POLLUTION}

\section{I. Principes généraux}

Certes, il est indispensable de protéger l'homme et la nature des effets des pollutions engendrées par les techniques industrielles, mais il est également nécessaire pour l'homme que le développement industriel soit poursuivi. Il faut donc faire en sorte que cette pollution soit maintenue dans des limites telles qu'elle ne soit pas nuisible, qu'elle ne soit pas agressive pour l'homme et son environnement. Cette notion de limite " tolérable ", " admissible », " acceptable », a fait l'objet de nombreuses discussions chez les radiobiologistes; elle n'en a pas moins été admise dans son esprit par tous. Cette limite peut être déterminée pour l'homme ou le milieu dans lequel il vit à partir de l'évaluation expérimentale chez l'animal, et conduit à l'établissement de normes qu'il s'agit alors de respecter et de faire respecter. Le contrôle rigoureux de la pollution consiste en somme à faire en sorte qu'elle ne puisse se transformer en agression.

\subsection{Protection radiologique}

Voyons dans quelle mesure ces principes généraux ont été mis en pratique dans le domaine de la protection contre les effets biologiques des rayonnements ionisants et de la protection contre la contamination radioactive.

Dans une remarquable analyse de cette protection de l'environnement présentée au $2^{\mathrm{e}}$ Congrès international de l'I.R.P.A., le Directeur de la Protection sanitaire de la Commission des Communautés Européennes, M. RECHr, a montré

vOL. $7-\mathrm{N}^{\mathrm{O}} 4$ 
que, dans le domaine nucléaire, les problèmes de protection ont toujours été étroitement associés au développement industriel et l'ont très souvent précédé.

L'évaluation des risques a fait l'objet de nombreuses recherches dans le domaine de la radiobiologie et la synthèse des travaux effectués, sur le plan mondial, a été faite par des commissions internationales compétentes qui ont également déterminé les normes à respecter pour l'irradiation externe et la contamination interne.

Je voudrais citer, à titre d'exemple, le rapport du Comité de la Commission Internationale de Protection Radiologique réalisé en 1965 et, en ce qui concerne la contamination, le rapport du Comité 2 de la C.I.P.R. concernant l'irradiation interne qui détermine les concentrations maximales admissibles dans l'eau et dans l'air, ainsi que les quantités maximales admissibles dans les organes critiques. Ce rapport datant de 1959 s'appuie sur I 771 publications, ce qui montre le sérieux avec lequel ce travail de compilation a été effectué.

Mais en ce qui concerne le milieu, la mesure des concentrations en radionucléides dans l'eau et dans l'air n'est pas toujours suffisante, en particulier lorsque la contamination se transmet par la chaîne alimentaire. Je voudrais alors à ce sujet vous renvoyer à l'excellent travail effectué par l'équipe de M. LAcourly, présenté au $2^{\mathrm{e}}$ Congrès international de l'I.R.P.A. sous le titre : "La protection radiologique des individus du public ». Dans sa communication, M. Lacourly définit la capacité d'acceptation du milieu pour un milieu quelconque de la façon suivante : « La capacité d'acceptation du milieu pour un polluant quelconque peut être définie comme la quantité de ce polluant que le milieu peut accepter d'une façon continue sans qu'il en résulte, à terme, de nuisances pour l'homme et son environnement. Elle s'exprime donc en poids, volume et activité par unité de temps m.

Il en découle que pour un polluant radioactif, la capacité d'acceptation s'exprimera en activité par unité de temps et qu'elle dépendra :

- de la dose limite d'irradiation par l'organe critique,

- des facteurs biologiques et nutritionnels de l'individu,

- des facteurs de transfert écologique milieu-homme,

- des facteurs intrinsèques du milieu récepteur qui déterminent, à l'équilibre, la concentration du radioélément dans ce milieu,

- de la forme physicochimique du radioélément.

Ces notions de dose limite pour l'organe critique et de capacité d'acceptation conduisent également à définir des valeurs relatives à l'ingestion limite pour l'individu et aux niveaux limites des aliments et du milieu vecteur.

Il faut tenir compte du fait que plusieurs radioéléments, plusieurs sources de pollution et plusieurs milieux récepteurs (eau, air, sol entre autres...) peuvent entrer en jeu dans le processus de contamination.

\subsection{Surveillance du milieu}

La fixation de normes, de doses limites, de niveaux admissibles de concentrations acceptables constitue la base indispensable à toute surveillance, celle-ci ne pouvant s'exercer que si la politique de protection est clairement définie. 
Et nous avons maints exemples, en France, de la rentabilité de cette politique dans le domaine nucléaire. Je ne voudrais citer que quelques exemples empruntés aux communications présentées à notre VIe Congrès international remarquablement organisé par M. CANDÈs et qui s'est tenu à Bordeaux du 27 au 30 mars 1972. MM. Chanteur et Moroni ont rappelé au cours de ce congrès que, conformément aux lois de 196 I et 1964 , les agents du Service central de la protection contre les rayonnements ionisants du Ministère de la Santé publique effectuent le contrôle des pollutions de tous ordres causées par les substances radioactives pour lesquels ils sont commissionnés et assermentés conformément aux dispositions du décret du is juin ig66. MM. Chanteur et Moroni pensent que ces lois doivent constituer le cadre fondamental d'un futur "Code de l'environnement" et disent, en conclusion de leur exposé intitulé " La réglementation française de radioprotection et son application ", que "Le développement industriel de l'énergie nucléaire a bénéficié de conditions telles que la pollution nucléaire a été, dès l'origine, convenablement maîtrisée et peut, sous bien des aspects, constituer un exemple de ce qu'il faudrait réaliser dans l'industrie conventionnelle ».

La communication de M. Marichal, qui avait trait au Contrôle de la pollution radioactive de l'environnement du Centre de Marcoule, montre avec quel soin sont effectués le contrôle atmosphérique des rejets des effluents gazeux radioactifs provenant du refroidissement des réacteurs, du traitement des combustibles irradiés et de la ventilation des locaux où sont manipulés des produits radioactifs, le contrôle systématique des végétaux du site de Marcoule, le contrôle des effluents liquides et des eaux du Rhône, le contrôle écologique de la nappe phréatique, des sédiments, de la faune et de la flore du Rhône. Les prélèvements étant analysés à la fois par le Service de protection radiologique du Centre de Marcoule et le Service central de protection contre les rayonnements ionisants.

MM. Martin et Beau du Département de protection de l'Electricité de France ont fait, également au Congrès de Bordeaux, le bilan de l'expérience acquise par l'E.D.F. dans le contrôle des rejets d'effluents des centrales nucléaires, tant au niveau de la source elle-même que de l'environnement. Les contrôles effectués par les services de l'E.D.F. et le S.C.P.R.I. ne mettent pas en évidence des contaminations anormales pendant le fonctionnement des diverses centrales.

Je n'ai volontairement pas parlé du contrôle et de la surveillance qui se sont exercés au cours des expérimentations nucléaires qui se sont déroulées au Sahara d'abord, dans le Pacifique ensuite. La radioprotection au cours des tirs nucléaires a montré le rôle éminent des techniciens de la radioprotection dans la protection de l'environnement, car c'est, en définitive, grâce à eux que ces essais ont pu être effectués dans des conditions telles qu'il n'en résulte aucun risque pour les populations.

Enfin, je voudrais citer les observations suivantes qu'il m'a été donné de lire récemment dans une publication médicale consacrée aux problèmes de pollution : “ Si la pollution atomique peut effectivement présenter de très grands dangers pour les êtres vivants, elle est la seule à avoir fait l'objet d'études préventives systématiques. Si bien que cette source de pollution semble être, dans la réalité des faits, réduite à l'extrême. Partout, des études très sérieuses ont précédé la construction des centres nucléaires et des conduites de rejets d'effluents. Partout, des contrôles très stricts sont effectués régulièrement. Partout, des stations poursuivent les recherches biologiques ou agricoles sur l'influence que pourrait

vOL. $7-\mathrm{N}^{\mathrm{O}} 4$ 
avoir le rejet des déchets, même très faiblement radioactifs, sur les organismes vivant dans l'eau où se font ces rejets et sur les cultures arrosées ou irriguées avec cette eau m.

\subsection{Protection contre les autres types de pollution}

Pour certains types de pollution non radioactive, des normes ont été établies avec précision et ont fait l'objet de textes réglementaires. On peut prendre, pour exemple entre autres, la pollution acoustique où l'on caractérise le bruit par son niveau d'intensité exprimé en décibels. Il est recommandé que l'intensité sonore soit inférieure à $35-45 \mathrm{~dB}$ à l'extérieur des habitations et $30 \mathrm{~dB}$ à l'intérieur. Le bruit dû à la circulation ne devrait pas dépasser $60 \mathrm{~dB}$, celui engendré par des machines $70 \mathrm{~dB}$, etc... mais, bien que ces normes, qui ne sont que des recommandations, soient bien connues, elles ne sont respectées que d'une manière imparfaite, car il est souvent difficile de modifier des constructions anciennes et il n'est pas toujours facile de mettre en œuvre une surveillance et un contrôle efficaces. J'ai emprunté ces chiffres à l'article de M. MATter de l'E.D.F. de la revue Agressologie.

Quant au problème de la pollution chimique, je me réfèrerai à un article du Professeur Truhaut paru en décembre i97I dans la revue officielle de la Fédération nationale de protection civile et j'extrairai de ses conclusions la citation suivante : « En ce qui concerne les produits toxiques pouvant se rencontrer dans l'atmosphère des locaux de travail, un des grands moyens de prévention contre leurs dangers est, après avoir fixé leurs seuils de toxicité, d'établir des limites de concentrations tolérables pour lesquelles et en deça desquelles, il n'y a pratiquement pas de dangers, soit pour des expositions de courte durée (valeurs plafonds), soit, lorsqu'il s'agit de poisons cumulatifs, pour des expositions répétées jour après jour, dans les conditions même du travail industriel ou agricole (valeurs moyennes intégrées par rapport au temps) ').

Le Professeur Truhaut indique également que ces limites tolérables doivent être également fixées pour les toxiques et leurs métabolites dans les matières biologiques (sang, urine, air expiré, etc...) dans l'air des locaux de travail, dans l'air des cités, et des environnements industriels, dans les denrées alimentaires, etc.

Et il conclut : «Il est hautement désirable de développer, à l'échelle mondiale, des centres de recherches toxicologiques convenablement équipés en matériel et en personnel qualifié pour appliquer les méthodes multidisciplinaires qu'il est nécessaire de mettre en œuvre dans l'étude des multiples problèmes actuels de la toxicologie. Nous ne saurons trop souligner à cet égard que, même dans les pays les plus à l'avant-garde comme les Etat-Unis par exemple, la toxicologie, science des poisons, reste encore un secteur sous développé. »)

On peut penser que dans ce domaine de la pollution chimique il reste beaucoup à faire.

\section{4 - CONCLUSIONS}

Les conclusions auxquelles on aboutit tout naturellement, lorsque l'on examine les divers problèmes relatifs à la pollution de l'environnement, ne peuvent être que celles dégagées par M. RECHT au cours de la séance de clôture du Sympo- 
sium international de la radioécologie appliquée à la protection de l'homme et de son environnement qui s'est tenu à Rome du 7 au ro septembre 1970 . M. RECHT pense qu'il est indispensable d'avoir une vision globale des problèmes de pollution, qu'il faut appliquer à la pollution non radioactive les principes et les méthodes en usage en radioécologie et en radioprotection et que, s'il n'est pas encore possible de définir une unité de nuisance analogue aux normes de base de la radioprotection, la notion de "dommage engagé " peut être utilisée pour définir des " critères et de nouveaux guides " en vue de l'établissement d'une réglementation relative aux polluants radioactifs.

Il serait souhaitable que l'établissement des critères de base pour les polluants non radioactifs soient établis par une autorité incontestée sur le plan international et, dans une communication présentée à ce même symposium, M. LAFONTAINE pense que l'O.M.S. pourrait jouer, dans ce domaine, un rôle analogue à celui de la C.I.P.R. dans celui de la pollution radioactive et préconise la création de groupe d'experts et de réseau de mesure dans le cadre de la Communauté Européenne.

Pour fixer la place de la contamination radioactive, je ne voudrais citer qu'une phrase de M. Lacourly qui est la suivante : "Il est heureux de constater que, dans la plupart des cas, la pollution radioactive reste à un niveau très bas par rapport à la capacité d'acceptation des milieux récepteurs, laissant ainsi de larges possibilités pour les installations futures. »

En ce qui concerne les pollutions non radioactives, il paraît urgent de se préoccuper de leurs effets et des moyens de s'en protéger. La " radioprotection " pourrait être le modèle de protection qu'il serait souhaitable de voir copier dans la majorité des cas de pollution, et tous ceux qui, au sein de notre Société, et bien que de disciplines diverses, œuvrent pour que cette radioprotection soit la meilleure possible sont prêts, j'en suis sûr, à faire bénéficier de leur expérience, de leur compétence, de leur technicité les hommes et les organismes qui feront appel à eux.

Je voudrais terminer cet exposé par une anecdote amusante empruntée à une chronique de Philippe ERLANGer : " des amateurs de beaujolais se plaignirent de ce que leur vin sentait le pétrole, ils avaient, en effet, lu qu'il était question d'installer une raffinerie dans la région où se trouvent les célèbres vignes et, comme disent les Anglais, ils avaient immédiatement sauté jusqu'aux conclusions "; mais, dit encore Philippe Erlanger : " les contemporains de Montaigne et de Descartes bravaient inconsciemment des périls dont l'idée seule nous rendrait malades comme la raffinerie fantôme gâta le goût du beaujolais dans la bouche d'un buveur obsédé ». Cette chronique avait pour titre : "Pollution sans obsession m.

Et cette anecdote me conduit à formuler ainsi ma conclusion qui aurait d'ailleurs pu être le titre de cet exposé :

POLLUTION sans AGRESSION ni OBSESSION grâce au modèle et aux techniciens de la RADIOPROTECTION 